\title{
Terahertz Imaging and Broadband Wireless Communication Using Plasma Oscillations in Nanometer Field Effect Transistors
}

\author{
W.Knap \\ Charles Coulomb Laboratory, Montpellier University and CNRS, France \\ $\&$ \\ High Pressure Institute, Polish Academy of Sciences Warsaw, Poland
}

\begin{abstract}
An overview of recent results concerning $\mathrm{THz}$ detection related to plasma nonlinearities in nanometer field effect transistors is presented. In particular nonlinearity and dynamic range of these detectors are discussed. We present also results on THz detection by Graphene field effect transistors. As a conclusion, we will show one of the first real world application of the FET THz detectors: a demonstrator of the imager developed for fast postal security imaging and wireless communication at carrier frequency above $300 \mathrm{GHz}$.
\end{abstract}

\section{INTRODUCTION}

We present an overview of some recent results concerning $\mathrm{THz}$ detection related to plasma nonlinearities in nanometer field effect transistors $[1,2]$. The subjects were selected in a way to show physics related limitations and advantages rather than purely technological or engineering improvements of nanometer Field Effect transistors (FETs) working as Terahertz detectors. We address the basic physics related problems like temperature dependence of the response [3], helicity sensitive detection [4] and nonlinear/saturation response at high incident power [5].

We present also the results on graphene based $\mathrm{THz}$ detectors [6-8] and first results on new $\mathrm{THz}$ detectors based on $\mathrm{Si}$ junction-less FETs [9]. The results will be discussed in view of the physical and technical limitations of Field Effect Transistors based $\mathrm{THz}$ detectors in view of their application for linear scanners [10].

\section{DYNAMIC RANGE}

Use Terahertz power dependence of the photoresponse of field effect transistors, operating at frequencies from 0.1 to 3 $\mathrm{THz}$ for incident radiation power density up to $100 \mathrm{~kW} / \mathrm{cm} 2$ was studied for Si metal-oxide-semiconductor field-effect transistors and InGaAs high electron mobility transistors.
The photoresponse increased linearly with increasing radiation intensity up to the $\mathrm{kW} / \mathrm{cm} 2$ range. Nonlinearity followed by saturation of the photoresponse was observed for all investigated field effect transistors for intensities above several $\mathrm{kW} / \mathrm{cm} 2$ - see Fig. 1. The observed photoresponse nonlinearity is explained by the saturation of the transistor channel current. The theoretical model of terahertz field effect transistor photoresponse at high intensity was developed. The model explains quantitatively experimental data both in linear and nonlinear (saturation) range. Our results show that dynamic range of field effect transistors is very high and can extend over more than six orders of magnitudes of power densities (from $\sim 0.5 \mathrm{~mW} / \mathrm{cm} 2$ to $\sim 5 \mathrm{~kW} / \mathrm{cm} 2$ ).

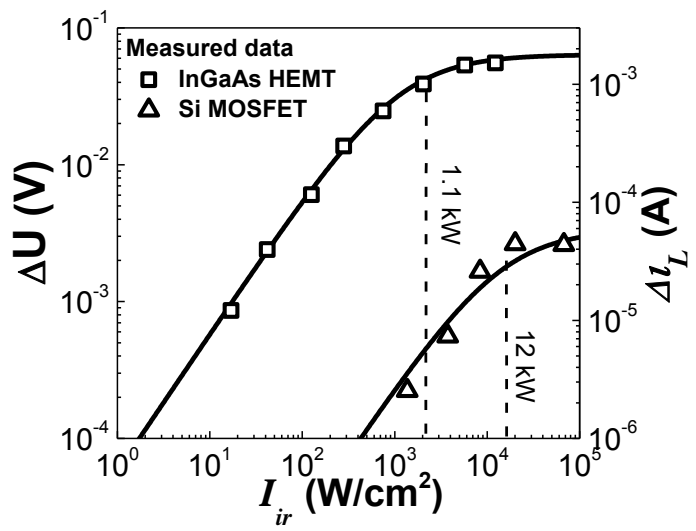

Fig. 1 Voltage and current generated by $\mathrm{THz}$ radiation. Saturation region is clearly visible. Solid lines are results of calculations [5]

\section{GRAPHENE PLASMA THZ DETECTORS}

Graphene, a one/two-atom-thick planar sheet of a honeycomb carbon crystal is a unique material with superior properties. The unusual gapless band structure of graphene 
with linear energy spectra for electrons and holes may lead to giant carrier mobility at room temperature and broadband flat optical response. In combination with huge thermal conductivity, these properties make graphene very appealing for electronics and sensor applications, including terahertz applications. Review of recent achievement on graphene based terahertz devices can be found in [11].

Exfoliated on $\mathrm{Si} / \mathrm{SO} 2$ single and double layer graphene flakes were used to fabricate top gate transistors. Log-periodic circular-toothed antennas at source and gate were used to couple $0.3 \mathrm{THz}$ radiation. A $35 \mathrm{~nm}$ thick $\mathrm{HfO} 2$ layer was used as the gate dielectric. The channel length was 7-10 $\mu \mathrm{m}$, while the gate length was $200-300 \mathrm{~nm}$. Fig. 2 shows the responsivity as a function of gate voltage. The specific feature of these dependencies is the change of the sign of the response at the charge neutrality (Dirac) voltage. Similar results were obtained at higher frequencies up to $3.11 \mathrm{THz}$ for back-gated graphene transistors [8].
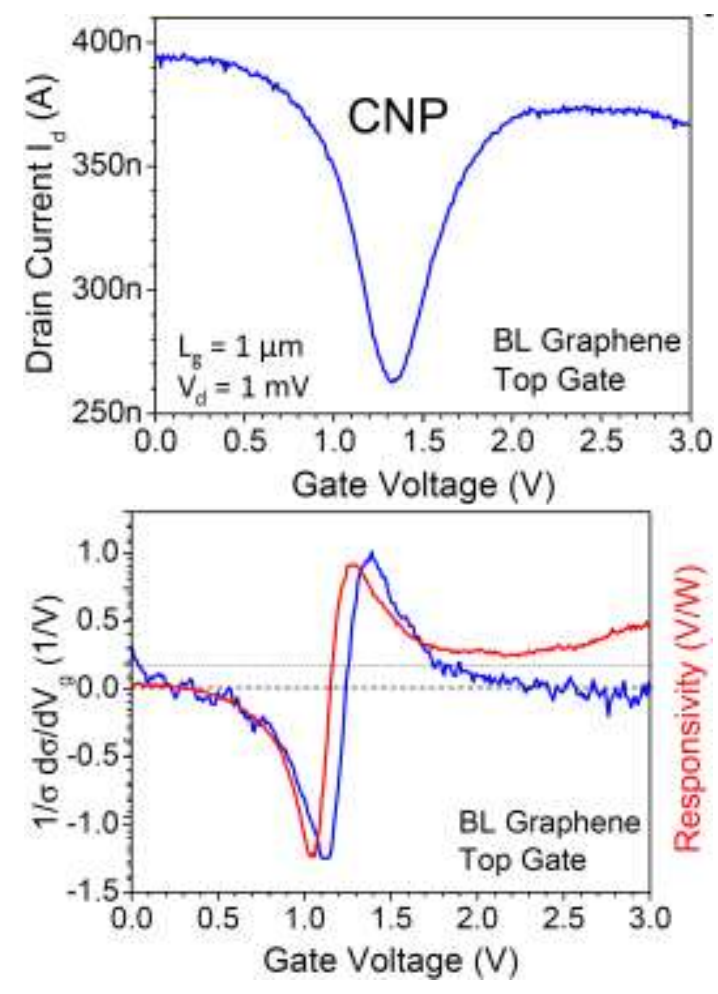

Fig. 2 The conductivity and responsivity as a function of gate voltage. The specific feature of these dependencies is the change of the sign of the $\mathrm{THz}$ response at the charge neutrality $(\mathrm{CNP})$ point $[6$, 7].

\section{DIFFRACTIVE OPTICS AND POSTAL SCANNER}

The active THz scanning systems present on the market require $\mathrm{THz}$ radiation source with specially adapted optics [24]. The first $\mathrm{THz}$ imaging systems were two axes raster scanning setups containing single point source and a single detector. They provided high quality images but the scanning time was relatively long, mainly limited by the speed of (XY) mechanical scanners. However, it appeared that to get the imaging speed acceptable for practical applications like postal security and on line nondestructive quality control so called linear scanners can be used. In these scanners the object moves on the transportation belt and the image is constructed line by line using linear multi-detector system. In principle the linear beam required in this case can be formed by a single rotating/vibrating mirror, but this imposes the speed limitations and long term mechanical stability of the system.

Recently diffractive elements that shape the illuminating divergent beam coming from the point-like source into a line segment in the given plane like shown in Fig. 3.

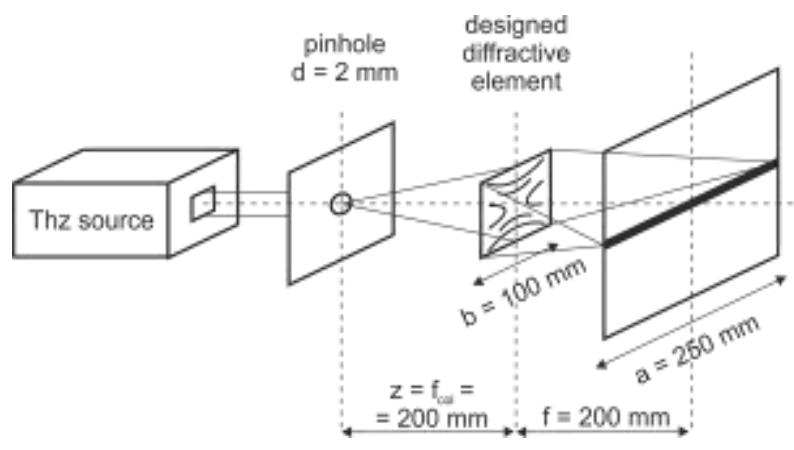

Fig. 3 Scheme of the optical system using diffractive elements that shape the illuminating divergent beam coming from the pointlike source into a line segment in the given plane [10].

In particular the structure based on the cost efficient 3D printing technology was demonstrated. The structure was experimentally evaluated and applied in a fast on line imaging system operating at $0.3 \mathrm{THz}$ atmospheric window, like shown in Fig 4. 


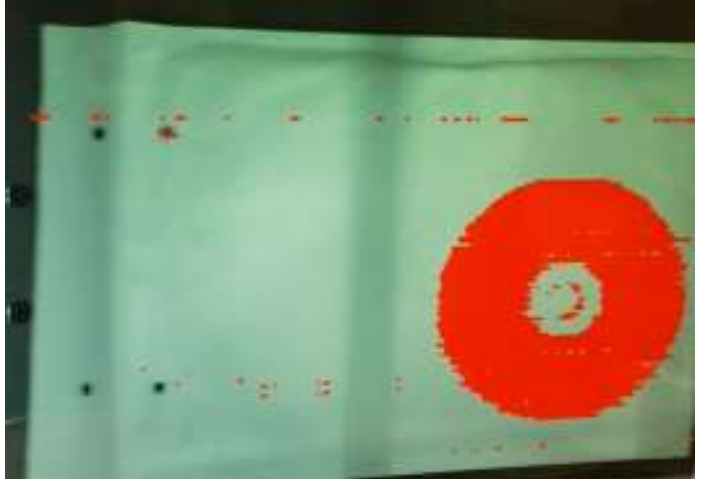

Fig. 4 The control monitor of the postal scanner. It shows a real time image of the envelope with $\mathrm{CD}$ disc inside. The envelope is moving on the control belt. Image is obtained by the fusion of the visible and $300 \mathrm{GHz}$ images [12].

\section{IV. $\mathrm{THz}$ wireless communication}

We would like to stress that both fast imaging and wireless communication require not only rectification of sub- $\mathrm{THz}$ signals with carrier frequency in the $300 / 600 \mathrm{GHz}$ band but also very high modulation speeds and/or wide band operation. Standard FET-based $\mathrm{THz}$ detectors operate at a subthreshold range that is characterized by the high channel resistance (order of MOhms). Therefore the RC constant (with parasitic capacitances) limits their speed/bandwidth.

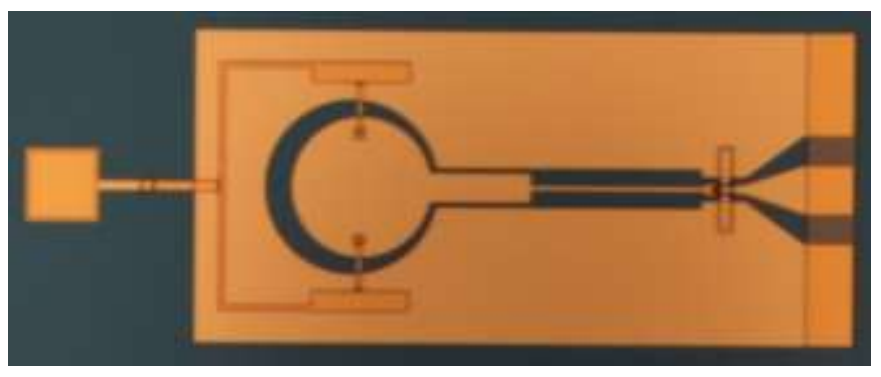

Fig. 5 Layout of plasma wave detector with slot ring antenna calculated for operation at $300 \mathrm{GHz}$. This detectors were successfully used in broadband wireless communication experiments $[13,14]$.

High modulation frequencies and wideband wireless communication require low channel resistance and therefore require special input /output impedance engineering. THz Wireless applications with plasma FET based receivers operating at sub-THz bands have been demonstrated, showing error free data rate up to 8.2 Gbits/s. $[13,14]$.

\section{ACKNOWLEDGEMENTS}

This work was partially supported by the National Centre for Research and Development in Poland (grant no. PBS1/A9/11/2012), by the National Science Centre in Poland (DEC-2013/10/M/ST3/00705) and by CNRS France via LIA -TERAMIR projects.

\section{REFERENCES}

[1] W. Knap and M. Dyakonov, in Handbook of Terahertz Technology edited by D. Saeedkia (Woodhead Publishing, Waterloo, Canada, 2013), pp. 121-155.

[2] W. Knap, S. Rumyantsev, M. Vitiello, D. Coquillat, S. Blin, N. Dyakonova, M. Shur, F. Teppe, A. Tredicucci and T. Nagatsuma, Nanotechnology 24 (21), 214002-214002 (2013).

[3] O. A. Klimenko, W. Knap, B. Iniguez, D. Coquillat, Y. A. Mityagin, F. Teppe, N. Dyakonova, H. Videlier, D. But, F. Lime, J. Marczewski and K. Kucharski, J. Appl. Phys. 112 (1), 014506-014505 (2012).

[4] C. Drexler, N. Dyakonova, P. Olbrich, J. Karch, M. Schafberger, K. Karpierz, Y. Mityagin, M. B. Lifshits, F. Teppe, O. Klimenko, Y. M. Meziani, W. Knap and S. D. Ganichev, J. Appl. Phys. 111 (12), 124504-124506 (2012).

[5] D. B. But, C. Drexler, M. V. Sakhno, N. Dyakonova, O. Drachenko, F. F. Sizov, A. Gutin, S. D. Ganichev and W. Knap, J. Appl. Phys. 115 (16), 164514 (2014).

[6] L. Vicarelli, M. S. Vitiello, D. Coquillat, A. Lombardo, A. C. Ferrari, W. Knap, M. Polini, V. Pellegrini and A. Tredicucci, Nat Mater 11 (10), 865-871 (2012).

[7] D. Spirito, D. Coquillat, S. L. De Bonis, A. Lombardo, M. Bruna, A. C. Ferrari, V. Pellegrini, A. Tredicucci, W. Knap and M. S. Vitiello, Appl. Phys. Lett. 104 (6), 061111 (2014).

[8] A. V. Muraviev, S. L. Rumyantsev, G. Liu, A. A. Balandin, W. Knap and M. S. Shur, Appl. Phys. Lett. 103 (18), 181114 (2013).

[9] J.Marczewski, W.Knap, D. Tomaszewski, M. Zaborowski, P. Zagrajek Silicon junction less FETs as room temperature $\mathrm{THz}$ detectors submitted to J. of Applied Phys - Jan. (2015)

[10] J. Suszek, A. Siemion, M. S. Bieda, N. Blocki, D. Coquillat, G. Cywinski, E. Czerwinska, M. Doch, A. Kowalczyk, N. Palka, A. Sobczyk, P. Zagrajek, M. Zaremba, A. Kolodziejczyk, W. Knap and M. Sypek, Terahertz Science and Technology, IEEE Transactions on 5 (2), 314-316 (2015).

[11] T. Otsuji, S. A. B. Tombet, A. Satou, H. Fukidome, M. Suemitsu, E. Sano, V. Popov, M. Ryzhii and V. Ryzhii, J. Phys. D:, Appl. Phys. 45 (30), 303001 (2012).

[12] Mail scanner: http://www.orteh.pl/page/22/research-development

[13] S. Blin, L. Tohme, D. Coquillat, S. Horiguchi, Y. Minamikata, S. Hisatake, P. Nouvel, T. Cohen, A. Penarier, F. Cano, L. Varani, W. Knap,' T. Nagatsuma, 'Wireless communication at $310 \mathrm{GHz}$ using GaAs high electron-mobility transistors for detection", Journal of Communications and Networks, vol. 15, no. 6 , p. 559, (2013).

[14] L. Tohme, G. Ducournau, S. Blin, D. Coquillat, P. Nouvel, A. Penarier, W. Knap, J. F. Lampin, ”0.2 THz wireless communication using plasma wave transistor detector", 38th International Conference on Infrared, Millimeter, and Terahertz Waves (IRMMW-THz) Mainz, Germany, Sep 2013, p. 1. 
of The Second Intl. Conf. On Advances In Computing, Control And Networking - ACCN 2015

Copyright $\odot$ Institute of Research Engineers and Doctors, USA .All rights reserved.

ISBN: 978-1-63248-073-6 doi: 10.15224/ 978-1-63248-073-6-82

Proof. W. Knap born in Poland in 1955 obtained his master degree from Faculty of Physics - Warsaw University Poland and got a permanent assistant professor position at Experimental Solid State Physics Department . His PhD concerned the Terahertz (Far infrared) properties of narrow gap semiconductors $\mathrm{HgTe}$ and $\mathrm{InSb}$. In 1987 he left to France and worked at University of Montpellier, Grenoble High Magnetic Field Laboratory, and Toulouse Pulsed High Magnetic Field Laboratory. In 1992 he obtained a permanent position at French National Centre for Scientific Research - CNRS - Montpellier where he works until now.. Between 1999 and 2001 he worked at- Rensellear Polytechnic Institute USA. He obtained also a long term grant from Japan Society of Promotion of Science and spent a year at Tohoku University 2007-2008. His main scientific interests are: i) Fair Infrared-FIR (Terahertz - THz) properties of semiconductors, ii) Quantum phenomena in transport and iii) Terahertz Plasma excitations in low dimensional structures. He is an author/coauthor of more than 100 publications in highly rated international journal and his citation $\mathrm{H}$ factor is higher than 35 .

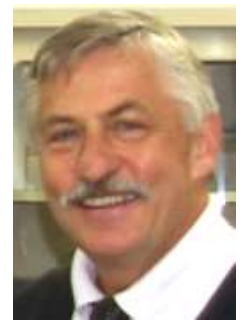

